\title{
Remarks concerning pair creation in strong magnetic fields
}

\author{
L. Semionova ${ }^{1}$ and D. Leahy ${ }^{2}$ \\ 1 Department of Physics, Universidad National, Heredia, Costa Rica 86-3000, Costa Rica \\ 2 Department of Physics \& Astronomy, University of Calgary, Alberta, Canada T2N 1N4, Canada
}

Received 20 September 2000 / Accepted 30 March 2001

\begin{abstract}
We derive the probability and attenuation coefficients for pair creation in first order of polarized electrons and positrons. The positron and electron can occupy arbitrary Landau levels and the attenuation coefficient is evaluated for any direction and polarization of the photon. When we take $\theta=90^{\circ}$ and sum over polarization of the spins of the $\mathrm{e}^{+}-\mathrm{e}^{-}$pair, we obtain results which differ from the expressions for the attenuation coefficient obtained by Daugherty \& Harding (1983), by the factor $\left(1+\delta_{N, 0}\right)\left(1+\delta_{N^{\prime}, 0}\right) / 4$, in agreement with Frangodimitraki-Georgiadou (1991).
\end{abstract}

Key words. quantum electrodynamics - magnetic fields - Landau levels - pair creation

\section{Introduction}

In this paper we analyse, in first order, the creation of an electron $\left(\mathrm{e}^{-}\right)$- positron $\left(\mathrm{e}^{+}\right)$pair in a strong uniform magnetic field. As was noted in Daugherty \& Harding (1983), quantum electrodynamic processes such as magnetic pair production and synchrotron radiation in the high field limit have come to play an important role in pulsar models. Also, one-photon pair production is likely to be the dominant source of $\mathrm{e}^{+}-\mathrm{e}^{-}$pairs in fields exceeding $10^{12}$ Gauss (Harding 1990). Daugherty \& Harding (1983) have a list of references relevant to this process. That paper presents analysis of the production of $\mathrm{e}^{+}-\mathrm{e}^{-}$pairs in fields exceeding $10^{12}$ Gauss for photon energies near threshold, and also for the limit of high photon energy. The energy distributions for the created pair is evaluated numerically near threshold and analytic expressions are given in the high photon energy limit. They find that as the field increases and as the photon energy increases, it is more probable that the photon energy is more unequally distributed between the $\mathrm{e}^{+}$and $\mathrm{e}^{-}$. Frangodimitraki-Georgiadou (1991) reanalyzed pair production in magnetic fields and obtained approximations for for total production rates in the limit of large photon energy for various values of the Landau levels of the created electron $(N)$ and positron $\left(N^{\prime}\right)$.

The purpose of this paper is to study the physical aspects of this elementary process of quantum electrodynamics, but generalizing from previous works by taking

Send offprint requests to: L. Semionova, e-mail: leahy@iras.ucalgary.ca into account the polarization of the electron and positron spins.

\section{Probability for pair creation}

The S-matrix element for a photon to interact with a magnetic field to produce an $\mathrm{e}^{+}-\mathrm{e}^{-}$pair may be written in the form:

$S_{\mathrm{fi}}=(-i \mathrm{e}) \int_{-\infty}^{\infty} \mathrm{d} t \int \mathrm{d}^{3} y \bar{\Psi}_{\mathrm{f}}(y) \gamma_{\mu} A^{\mu}(y) \Psi_{\mathrm{i}}(y)$

with $A^{\mu}(y, k)=\epsilon^{\mu} /(2 \omega V)^{1 / 2} \mathrm{e}^{-i k y}$, and $\Psi_{\mathrm{f}}(y)$ and $\Psi_{\mathrm{i}}(y)$ are the wave functions for electron and positron, respectively. We use the wave functions for $\mathrm{e}^{-}$and $\mathrm{e}^{+}$defined by Sokolov \& Ternov (1983).

The photon has four possible polarizations to consider. The two linear polarizations are defined by the unit vectors:

$$
\begin{aligned}
& \hat{\varepsilon}^{(1)}=-\cos \theta \cos \varphi \cdot \hat{i}-\cos \theta \sin \varphi \cdot \hat{j}+\sin \theta \cdot \hat{k} \\
& \hat{\varepsilon}^{(2)}=\sin \varphi \cdot \hat{i}-\cos \varphi \cdot \hat{j}
\end{aligned}
$$

with $\theta, \varphi$ the spherical polar angular coordinates of the photon. The photon momentum vector is given by:

$\boldsymbol{k}=\omega(\sin \theta \cos \varphi \cdot \hat{i}+\sin \theta \sin \varphi \cdot \hat{j}+\cos \theta \cdot \hat{k})$.

The unit vectors $\hat{\varepsilon}^{(1)}, \hat{\varepsilon}^{(2)}$ and $\hat{k}$ form a right handed triad:

$\hat{\varepsilon}^{(1)} \times \hat{\varepsilon}^{(2)}=\hat{k}$.

Also it is easy to show that these two polarizations, namely $\perp\left(\hat{\varepsilon}^{(2)}\right)$ and $\|\left(\hat{\varepsilon}^{(1)}\right)$, have the photon's electric field vector 
respectively parallel or perpendicular to $\boldsymbol{k} \times \boldsymbol{B}$ (Baring \& Harding 1995). The labels $\perp$ and $\|$ are due to the property of being respectively perpendicular or parallel to $\boldsymbol{B} . \hat{\varepsilon}^{(2)}$ is perpendicular to $\boldsymbol{B}$ independent of $\theta$, but $\hat{\varepsilon}^{(1)}$ is parallel to $\boldsymbol{B}$ only if $\theta=\pi / 2$. Generally, the angle between $\hat{\varepsilon}^{(1)}$ and $\boldsymbol{B}$ is:

$\theta^{\prime}=\sin ^{-1}(\cos (\theta))$.

Right and left circular polarization states are obtained by the linear combinations:

$\hat{\varepsilon}_{ \pm}=\mp \frac{1}{\sqrt{2}}\left(\hat{\varepsilon}^{(1)} \pm i \hat{\varepsilon}^{(2)}\right)$.

The energies for $\mathrm{e}^{-}(E)$ and $\mathrm{e}^{+}\left(E^{\prime}\right)$ are:

$E=\left[m^{2}+p_{z}{ }^{2}+2 m^{2} B^{\prime} N\right]^{1 / 2}$

$E^{\prime}=\left[m^{2}+p_{z}^{\prime 2}+2 m^{2} B^{\prime} N^{\prime}\right]^{1 / 2}$

or, in units of $m$ :

$$
\begin{aligned}
& E=\left[1+p_{z}{ }^{2}+2 B^{\prime} N\right]^{1 / 2} \\
& E^{\prime}=\left[1+p_{z}^{\prime 2}+2 B^{\prime} N^{\prime}\right]^{1 / 2} .
\end{aligned}
$$

Here $p_{z}, p_{z}^{\prime}$ are the longitudinal momenta for $\mathrm{e}^{-}$and $\mathrm{e}^{+}$, respectively; $N, N^{\prime}=0,1,2, \ldots$ are the Landau levels of $\mathrm{e}^{-}$ and $\mathrm{e}^{+}$; and $B^{\prime}=B / B_{\mathrm{cr}}$, with $B_{\mathrm{cr}}=m^{2} c^{3} /(\mathrm{e} \hbar)=$ $4.414 \times 10^{13}$ Gauss. We use natural units hereafter in which $c=\hbar=1$. The Landau quantum number is $N=n+(\sigma+1) / 2$, where $\sigma= \pm 1$ may be interpreted as the spin eigenvalue, and $n=0,1,2, \ldots$ Excited states are degenerate yet the ground state, $N=0$ or $N^{\prime}=0$, is non-degenerate for both particles: for $\mathrm{e}^{-}$, the spin is opposite to the direction of the magnetic field $(\sigma=-1)$; for $\mathrm{e}^{+}$, the spin is parallel to the direction of the magnetic field $(\sigma=+1)$ (e.g. Frangodimitraki-Georgiadou 1991).

The probability for the creation of pairs in the general case, where $r, r^{\prime}$ is the polarization of $\mathrm{e}^{-}$and $\mathrm{e}^{+}$, respectively, is:

$$
\begin{aligned}
\left|S_{\mathrm{fi}}\right|^{2}= & \frac{\mathrm{e}^{2}}{2 \omega V} \frac{1}{L^{2}}\left[2 \pi \delta\left(E+E^{\prime}-\omega\right)\right]^{2}\left[2 \pi \delta\left(p_{z}+p_{z}{ }^{\prime}-\omega_{z}\right)\right]^{2} \\
& \times \frac{1}{8 E E^{\prime} E_{\mathrm{o}} E_{\mathrm{o}}^{\prime}} I_{s, s^{\prime}}{ }^{2}\left(\frac{\omega^{2} \sin ^{2} \theta}{2 \mathrm{e} B}\right)\left[N_{\mathrm{p}}\right]
\end{aligned}
$$

with:

$$
\begin{aligned}
& {\left[N_{\mathrm{p}}\right]=\varepsilon^{(\lambda)}{ }_{z} \varepsilon^{(\lambda)}{ }_{z}{ }^{*}\left(E^{\prime} E+p_{z}{ }^{\prime} p_{z}-r r^{\prime} E_{\mathrm{o}}{ }^{\prime} E_{\mathrm{o}}\right)} \\
& \times\left[\left(E_{\mathrm{o}}{ }^{\prime}-r^{\prime} m\right)\left(E_{\mathrm{o}}+r m\right) I_{N^{\prime}-1, N-1}{ }^{2}\right. \\
& \left.+\left(E_{\mathrm{o}}{ }^{\prime}+r^{\prime} m\right)\left(E_{\mathrm{o}}-r m\right) I_{N^{\prime}, N^{2}}{ }^{2}\right] \\
& -\left(E_{\mathrm{o}}+r m\right) \sqrt{2 \mathrm{e} B N^{\prime}}\left(p_{z} E_{\mathrm{o}}{ }^{\prime}+r r^{\prime} p_{z}{ }^{\prime} E_{\mathrm{O}}\right) \\
& \times I_{N^{\prime}-1, N-1} I_{N^{\prime}, N-1} \\
& \times\left[\varepsilon^{(\lambda)} z^{(\lambda)}{ }^{*} \mathrm{e}^{-i \varphi}+\varepsilon^{(\lambda)}-\varepsilon^{(\lambda)}{ }_{z}^{*} \mathrm{e}^{i \varphi}\right] \\
& -r r^{\prime} \sqrt{2 \mathrm{e} B N}\left(p_{z} E_{\mathrm{o}}{ }^{\prime}+r r^{\prime} p_{z}{ }^{\prime} E_{\mathrm{o}}\right)\left(E_{\mathrm{o}}{ }^{\prime}-r^{\prime} m\right) \\
& \times I_{N^{\prime}-1, N-1} I_{N^{\prime}-1, N} \\
& \times\left[\varepsilon^{(\lambda)} \varepsilon^{(\lambda)}+{ }^{*} \mathrm{e}^{i \varphi}+\varepsilon^{(\lambda)}+\varepsilon^{(\lambda)} z^{*} \mathrm{e}^{-i \varphi}\right] \\
& -\varepsilon^{(\lambda)} \varepsilon^{(\lambda)}{ }_{z}^{*} 4 \mathrm{e} B \sqrt{N N^{\prime}}
\end{aligned}
$$

$$
\begin{aligned}
& \times\left(-r r^{\prime} E^{\prime} E-r r^{\prime} p_{z}{ }^{\prime} p_{z}+E_{\mathrm{o}}{ }^{\prime} E_{\mathrm{O}}\right) I_{N^{\prime}-1, N-1} I_{N^{\prime}, N} \\
& +\left(E^{\prime} E-p_{z}{ }^{\prime} p_{z}+r r^{\prime} E_{\mathrm{O}}{ }^{\prime} E_{\mathrm{O}}\right) \\
& \times\left[\varepsilon^{(\lambda)}-\varepsilon^{(\lambda)}-{ }^{*}\left(E_{\mathrm{O}}{ }^{\prime}+r^{\prime} m\right)\left(E_{\mathrm{O}}+r m\right) I_{N^{\prime}, N-1}{ }^{2}\right. \\
& \left.+\varepsilon^{(\lambda)}+\varepsilon^{(\lambda)}+{ }^{*}\left(E_{\mathrm{O}}-r m\right)\left(E_{\mathrm{O}}{ }^{\prime}-r^{\prime} m\right) I_{N^{\prime}-1, N}{ }^{2}\right] \\
& +2 e B \sqrt{N N^{\prime}}\left(r r^{\prime} E^{\prime} E-r r^{\prime} p_{z}{ }^{\prime} p_{z}+E_{\mathrm{O}}{ }^{\prime} E_{\mathrm{O}}\right) \\
& \times I_{N^{\prime}, N-1} I_{N^{\prime}-1, N}\left[\varepsilon^{(\lambda)}{ }_{-} \varepsilon^{(\lambda)}+{ }^{*} \mathrm{e}^{i 2 \varphi}+\varepsilon^{(\lambda)}\right. \\
& \left.+\varepsilon^{(\lambda)}-{ }^{*} \mathrm{e}^{-i 2 \varphi}\right]-r r^{\prime} \sqrt{2 \mathrm{e} B N}\left(E_{\mathrm{o}}{ }^{\prime}+r^{\prime} m\right) \\
& \times\left(p_{z} E_{\mathrm{o}}{ }^{\prime}+r r^{\prime} p_{z}{ }^{\prime} E_{\mathrm{O}}\right) I_{N^{\prime}, N-1} I_{N^{\prime}, N} \\
& \times\left[\varepsilon^{(\lambda)}-\varepsilon^{(\lambda)}{ }_{z}^{*} \mathrm{e}^{i \varphi}+\varepsilon^{(\lambda)} \varepsilon^{(\lambda)}-{ }^{*} \mathrm{e}^{-i \varphi}\right] \\
& -\left(E_{\mathrm{O}}-r m\right) \sqrt{2 \mathrm{e} B N^{\prime}}\left(p_{z} E_{\mathrm{O}}{ }^{\prime}+r r^{\prime} p_{z}{ }^{\prime} E_{\mathrm{O}}\right) \\
& \times I_{N^{\prime}-1, N} I_{N^{\prime}, N}\left[\varepsilon^{(\lambda)}+\varepsilon^{(\lambda)}{ }_{z}^{*} \mathrm{e}^{-i \varphi}\right. \\
& \left.\left.+\varepsilon^{(\lambda)} \varepsilon^{(\lambda)}+{ }^{*} \mathrm{e}^{i \varphi}\right)\right] \text {. }
\end{aligned}
$$

In the above the argument $x$ of the Laguerre function $I_{N^{\prime}, N}(x)$ is $\frac{\omega^{2} \sin ^{2} \theta}{2 \mathrm{e} B} ; E_{\mathrm{o}}=\left(m^{2}+2 N \mathrm{e} B\right)^{1 / 2}, E_{\mathrm{o}}{ }^{\prime}=\left(m^{2}+\right.$ $\left.2 N^{\prime} \mathrm{e} B\right)^{1 / 2}$; and and $\varepsilon^{(\lambda)}{ }_{ \pm}=\varepsilon^{(\lambda)}{ }_{x} \pm i \varepsilon^{(\lambda)}{ }_{y}$.

Averaging over the polarization of the positron in Eq. (11), we obtain the probability for pair creation of a polarized electron $(r= \pm 1)$, with any polarization for the positron:

$$
\begin{aligned}
\left|S_{\mathrm{fi}}\right|^{2} & =\frac{\mathrm{e}^{2}}{2 \omega V} \frac{1}{L^{2}}\left[2 \pi \delta\left(E+E^{\prime}-\omega\right)\right]^{2}\left[2 \pi \delta\left(p_{z}+p_{z}{ }^{\prime}-\omega_{z}\right)\right]^{2} \\
& \times \frac{1}{4 E E^{\prime} E_{\mathrm{o}}} I_{s, s^{\prime}} 2\left(\frac{\omega^{2} \sin ^{2} \theta}{2 e B}\right) \frac{\left(1+\delta_{N^{\prime}, 0}\right)}{2}\left[N_{\mathrm{p}}\right]_{1}
\end{aligned}
$$

with:

$$
\begin{aligned}
& {\left[N_{\mathrm{p}}\right]_{1}=\varepsilon^{(\lambda)}{ }_{z} \varepsilon^{(\lambda)}{ }_{z}{ }^{*}\left[\left(E_{\mathrm{O}}+r m\right)\left(E^{\prime} E+p_{z}{ }^{\prime} p_{z}+r E_{\mathrm{o}} m\right)\right.} \\
& \times I_{N^{\prime}-1, N-1}^{2}+\left(E_{\mathrm{O}}-r m\right) \\
& \left.\times\left(E^{\prime} E+p_{z}{ }^{\prime} p_{z}-r E_{\mathrm{o}} m\right) I_{N^{\prime}, N}{ }^{2}\right]-p_{z}\left(E_{\mathrm{o}}+r m\right) \\
& \times \sqrt{2 \mathrm{e} B N^{\prime}} I_{N^{\prime}-1, N-1} I_{N^{\prime}, N-1} \\
& \times\left[\varepsilon^{(\lambda)}{ }_{z} \varepsilon^{(\lambda)^{*}}{ }_{-} \mathrm{e}^{-i \varphi}+\varepsilon^{(\lambda)}{ }_{-} \varepsilon^{(\lambda)}{ }_{z}^{*} \mathrm{e}^{i \varphi}\right] \\
& -\sqrt{2 \mathrm{e} B N} r\left(r p_{z}{ }^{\prime} E_{\mathrm{o}}-m p_{z}\right) \\
& { }_{N^{\prime}-1, N-1} I_{N^{\prime}-1, N}\left[\varepsilon^{(\lambda)} \varepsilon^{(\lambda)}+{ }^{*} \mathrm{e}^{i \varphi}\right. \\
& \left.+\varepsilon^{(\lambda)}+\varepsilon^{(\lambda)}{ }_{z}^{*} \mathrm{e}^{-i \varphi}\right] \\
& -4 \mathrm{e} B \sqrt{N N^{\prime}} \varepsilon^{(\lambda)}{ }_{z} \varepsilon^{(\lambda)}{ }_{z}{ }^{*} E_{\mathrm{o}} I_{N^{\prime}-1, N-1} I_{N^{\prime}, N} \\
& +\left[\left(E_{\mathrm{o}}+r m\right)\left(E^{\prime} E-p_{z}{ }^{\prime} p_{z}+r E_{\mathrm{o}} m\right)\right. \\
& \times I_{N^{\prime}, N-1}{ }^{2} \varepsilon^{(\lambda)} \varepsilon^{(\lambda)}{ }_{-}^{*} \\
& +\left(E_{\mathrm{o}}-r m\right)\left(E^{\prime} E-p_{z}^{\prime} p_{z}-r E_{\mathrm{o}} m\right) \varepsilon^{(\lambda)}+ \\
& \left.\varepsilon^{(\lambda)}{ }_{+}^{*} I_{N^{\prime}-1, N}{ }^{2}\right]+2 \mathrm{e} B \sqrt{N N^{\prime}} E_{\mathrm{o}} \\
& \times I_{N^{\prime}, N-1} I_{N^{\prime}-1, N} \\
& {\left[\varepsilon^{(\lambda)}-\varepsilon^{(\lambda)}+{ }^{*} \mathrm{e}^{i 2 \varphi}+\varepsilon^{(\lambda)}+\varepsilon^{(\lambda)}{ }^{*} \mathrm{e}^{-i 2 \varphi}\right]} \\
& -r \sqrt{2 \mathrm{e} B N}\left(r p_{z}{ }^{\prime} E_{\mathrm{O}}+m p_{z}\right) \\
& \times I_{N^{\prime}, N-1} I_{N^{\prime}, N}\left[\varepsilon^{(\lambda)}-\varepsilon^{(\lambda)} z^{*} \mathrm{e}^{i \varphi}+\varepsilon^{(\lambda)} z^{\varepsilon^{(\lambda)}}{ }_{-}^{*} \mathrm{e}^{-i \varphi}\right] \\
& -p_{z}\left(E_{\mathrm{o}}-r m\right) \sqrt{2 \mathrm{e} B N^{\prime}} \\
& \left.\times I_{N^{\prime}-1, N} I_{N^{\prime}, N}\left[\varepsilon^{(\lambda)}+\varepsilon^{(\lambda)}{ }_{z}^{*} \mathrm{e}^{-i \varphi}+\varepsilon^{(\lambda)}{ }_{z} \varepsilon^{(\lambda)}+{ }^{*} \mathrm{e}^{i \varphi}\right)\right] \text {. }
\end{aligned}
$$


Averaging over the polarization of the electron in Eq. (11), we obtain the probability for pair creation of a polarized positron $\left(r^{\prime}= \pm 1\right)$, with any polarization for the electron:

$$
\begin{aligned}
\left|S_{\mathrm{fi}}\right|^{2}= & \frac{\mathrm{e}^{2}}{2 \omega V} \frac{1}{L^{2}}\left[2 \pi \delta\left(E+E^{\prime}-\omega\right)\right]^{2}\left[2 \pi \delta\left(p_{z}+p_{z}{ }^{\prime}-\omega_{z}\right)\right]^{2} \\
& \times \frac{1}{4 E E^{\prime} E_{\mathrm{o}}^{\prime}} I_{s, s^{\prime}}{ }^{2}\left(\frac{\omega^{2} \sin ^{2} \theta}{2 e B}\right) \frac{\left(1+\delta_{N, 0}\right)}{2}\left[N_{\mathrm{p}}\right]_{2}(13)
\end{aligned}
$$

with:

$$
\begin{aligned}
& {\left[N_{\mathrm{p}}\right]_{2}=\varepsilon^{(\lambda)}{ }_{z} \varepsilon^{(\lambda)}{ }_{z}{ }^{*}\left[\left(E_{\mathrm{o}}{ }^{\prime}-r^{\prime} m\right)\left(E^{\prime} E+p_{z}{ }^{\prime} p_{z}-r^{\prime} E_{\mathrm{o}}{ }^{\prime} m\right)\right.} \\
& \times I_{N^{\prime}-1, N-1}{ }^{2}+\left(E_{\mathrm{o}}{ }^{\prime}+r^{\prime} m\right)\left(E^{\prime} E+p_{z}{ }^{\prime} p_{z}\right. \\
& \left.\left.+r^{\prime} E_{\mathrm{o}}{ }^{\prime} m\right) I_{N^{\prime}, N}{ }^{2}\right]-\left(p_{z} E_{\mathrm{o}}{ }^{\prime}+r^{\prime} m p_{z}{ }^{\prime}\right) \\
& \times \sqrt{2 \mathrm{e} B N^{\prime}} I_{N^{\prime}-1, N-1} I_{N^{\prime}, N-1} \\
& \times\left[\varepsilon^{(\lambda)} z \varepsilon^{(\lambda)}-{ }^{*} \mathrm{e}^{-i \varphi}+\varepsilon^{(\lambda)}-\varepsilon^{(\lambda)} z^{*} \mathrm{e}^{i \varphi}\right] \\
& -p_{z}{ }^{\prime}\left(E_{\mathrm{o}}{ }^{\prime}-r^{\prime} m\right) \sqrt{2 \mathrm{e} B N} I_{N^{\prime}-1, N-1} I_{N^{\prime}-1, N} \\
& \times\left[\varepsilon^{(\lambda)}{ }_{z} \varepsilon^{(\lambda)}+{ }^{*} \mathrm{e}^{i \varphi}+\varepsilon^{(\lambda)}+\varepsilon^{(\lambda)} z^{*} \mathrm{e}^{-i \varphi}\right] \\
& -\varepsilon^{(\lambda)}{ }_{z} \varepsilon^{(\lambda)}{ }_{z}{ }^{*} 4 \mathrm{e} B \sqrt{N N^{\prime}} E_{\mathrm{O}}{ }^{\prime} I_{N^{\prime}-1, N-1} I_{N^{\prime}, N} \\
& +\left[\varepsilon^{(\lambda)}-\varepsilon^{(\lambda)}{ }^{*}\left(E_{\mathrm{o}}{ }^{\prime} r^{\prime} m\right)\left(E^{\prime} E-p_{z}^{\prime} p_{z}+r^{\prime} E_{\mathrm{O}}{ }^{\prime} m\right)\right. \\
& \times I_{N^{\prime}, N-1}{ }^{2}+\varepsilon^{(\lambda)}+\varepsilon^{(\lambda)}+{ }^{*}\left(E_{\mathrm{o}}{ }^{\prime}-r^{\prime} m\right)\left(E^{\prime} E\right. \\
& \left.-p_{z}{ }^{\prime} p_{z}-r^{\prime} E_{\mathrm{o}}{ }^{\prime} m\right) \\
& \left.\times I_{N^{\prime}-1, N^{2}}{ }^{2}\right]+2 \mathrm{e} B \sqrt{N N^{\prime}} E_{\mathrm{O}}{ }^{\prime} I_{N^{\prime}, N-1} \\
& \times I_{N^{\prime}-1, N}\left[\varepsilon^{(\lambda)}-\varepsilon^{(\lambda)}+{ }^{*} \mathrm{e}^{i 2 \varphi}+\varepsilon^{(\lambda)}+\varepsilon^{(\lambda)}-{ }^{*} \mathrm{e}^{-i 2 \varphi}\right] \\
& -\sqrt{2 \mathrm{e} B N} p_{z}{ }^{\prime}\left(E_{\mathrm{O}}{ }^{\prime}+r^{\prime} m\right) I_{N^{\prime}, N-1} I_{N^{\prime}, N} \\
& \times\left[\varepsilon^{(\lambda)}-\varepsilon^{(\lambda)} z^{*} \mathrm{e}^{i \varphi}+\varepsilon^{(\lambda)}{ }_{z} \varepsilon^{(\lambda)}-{ }^{*} \mathrm{e}^{-i \varphi}\right] \\
& -\sqrt{2 \mathrm{e} B N^{\prime}}\left(p_{z} E_{\mathrm{o}}{ }^{\prime}-r^{\prime} m p_{z}{ }^{\prime}\right) I_{N^{\prime}-1, N} I_{N^{\prime}, N} \\
& \left.\times\left[\varepsilon^{(\lambda)}+\varepsilon^{(\lambda)} z^{*} \mathrm{e}^{-i \varphi}+\varepsilon^{(\lambda)} \varepsilon^{(\lambda)}+{ }^{*} \mathrm{e}^{i \varphi}\right)\right] .
\end{aligned}
$$

By taking the average over the polarization of the electron in the above, we obtain the probability for pair creation of an electron-positron pair with any polarization:

$$
\begin{aligned}
\left|S_{\mathrm{fi}}\right|^{2}= & \frac{\mathrm{e}^{2}}{2 \omega V} \frac{1}{L^{2}}\left[2 \pi \delta\left(E+E^{\prime}-\omega\right)\right]^{2}\left[2 \pi \delta\left(p_{z}+p_{z}{ }^{\prime}-\omega_{z}\right)\right]^{2} \\
& \times \frac{1}{2 E E^{\prime}} I_{s, s^{\prime}}{ }^{2}\left(\frac{\omega^{2} \sin ^{2} \theta}{2 \mathrm{e} B}\right) \\
& \times \frac{\left(1+\delta_{N, 0}\right)\left(1+\delta_{N^{\prime}, 0}\right)}{4}\left[N_{\mathrm{p}}\right]_{3}
\end{aligned}
$$

with:

$$
\begin{aligned}
{\left[N_{\mathrm{p}}\right]_{3}=} & \varepsilon^{(\lambda)}{ }_{z} \varepsilon^{(\lambda)}{ }_{z}^{*}\left(E^{\prime} E+p_{z}{ }^{\prime} p_{z}+m^{2}\right) \\
& \times\left(I_{N^{\prime}-1, N-1}{ }^{2}+I_{N^{\prime}, N^{2}}\right)-p_{z} \sqrt{2 \mathrm{e} B N^{\prime}} I_{N^{\prime}-1, N-1} \\
& \times I_{N^{\prime}, N-1}\left[\varepsilon^{(\lambda)} \varepsilon \varepsilon^{(\lambda)}-{ }^{*} \mathrm{e}^{-i \varphi}+\varepsilon^{(\lambda)}-\varepsilon^{(\lambda)} z^{*} \mathrm{e}^{i \varphi}\right] \\
& -p_{z}{ }^{\prime} \sqrt{2 \mathrm{e} B N} I_{N^{\prime}-1, N-1} I_{N^{\prime}-1, N} \\
& \times\left[\varepsilon^{(\lambda)} \varepsilon^{(\lambda)}+{ }^{*} \mathrm{e}^{i \varphi}+\varepsilon^{(\lambda)}+\varepsilon^{(\lambda)} z^{*} \mathrm{e}^{-i \varphi}\right] \\
& -4 \mathrm{e} B \sqrt{N N^{\prime}} \varepsilon^{(\lambda)} \varepsilon^{(\lambda)} z^{*} I_{N^{\prime}-1, N-1} I_{N^{\prime}, N} \\
& +\left(E^{\prime} E-p_{z}{ }^{\prime} p_{z}+m^{2}\right)\left[\varepsilon^{(\lambda)}-\varepsilon^{(\lambda)}-{ }^{*} I_{N^{\prime}, N-1}{ }^{2}\right. \\
& \left.+\varepsilon^{(\lambda)}+\varepsilon^{(\lambda)}+{ }^{*} I_{N^{\prime}-1, N}{ }^{2}\right]+2 \mathrm{e} B \sqrt{N N^{\prime}} I_{N^{\prime}, N-1} \\
& \times I_{N^{\prime}-1, N}\left[\varepsilon^{(\lambda)}-\varepsilon^{(\lambda)}+\mathrm{e}^{*}+\varepsilon^{(\lambda)}+\varepsilon^{(\lambda)}-\mathrm{e}^{-i 2 \varphi}\right]
\end{aligned}
$$

$$
\begin{aligned}
& -\sqrt{2 \mathrm{e} B N} p_{z}{ }^{\prime} I_{N^{\prime}, N-1} I_{N^{\prime}, N}\left[\varepsilon^{(\lambda)} \varepsilon^{(\lambda)} z^{*} \mathrm{e}^{i \varphi}\right. \\
& \left.\left.+\varepsilon^{(\lambda)} \varepsilon^{(\lambda)}-\mathrm{e}^{-i \varphi}\right)\right] \\
& -\sqrt{2 \mathrm{e} B N^{\prime}} p_{z} I_{N^{\prime}-1, N} I_{N^{\prime}, N}\left[\varepsilon^{(\lambda)}+\varepsilon^{(\lambda)}{ }_{z}^{*} \mathrm{e}^{-i \varphi}\right. \\
& \left.+\varepsilon^{(\lambda)}{ }_{z} \varepsilon^{(\lambda)}+\mathrm{e}^{i \varphi}\right]
\end{aligned}
$$

$I_{N, R}$ is the Laguerre function, defined as:

$I_{N, R}(x)=\sqrt{R ! / N !} \mathrm{e}^{-x / 2} x^{(N-R) / 2} L_{R}^{N-R}(x)$.

Summing over radial quantum number $s^{\prime}$ yields:

$\sum_{s^{\prime}=0}^{\infty} I_{s, s^{\prime}}{ }^{2}(x)=1$.

However, the sum over $s$ is limited to some maximum value $s_{\max }$ (see Sokolov \& Ternov 1983), so

$\sum_{s=0}^{s_{\max }} \sum_{s^{\prime}=0}^{\infty}{I_{s, s^{\prime}}}^{2}\left(\omega_{\perp}{ }^{2} / 2 \mathrm{e} B\right)=\frac{m^{2} B^{\prime}}{2 \pi} L^{2}$

with $\omega_{\perp}=\omega \sin \theta$.

We obtain two laws of conservation for energy and third component of momentum:

$p_{z}+p_{z}{ }^{\prime}=\omega_{z} \quad E+E^{\prime}=\omega$.

The following properties are also used:

$$
\begin{aligned}
& {\left[\delta\left(p_{z}+p_{z}{ }^{\prime}-\omega_{z}\right)\right]^{2}=\frac{L_{z}}{2 \pi} \delta\left(p_{z}+p_{z}{ }^{\prime}-\omega_{z}\right)} \\
& {\left[\delta\left(E+E^{\prime}-\omega\right)\right]^{2}=\frac{T}{2 \pi} \delta\left(E+E^{\prime}-\omega\right) .}
\end{aligned}
$$

Then, averaging over the two independent polarizations states of the photon, the probability for pair creation for polarized electron and positron is:

$$
\begin{aligned}
& \left|S_{\mathrm{fi}}\right|^{2}=\frac{\mathrm{e}^{2}}{4 \omega V} \frac{1}{L^{2}}\left[2 \pi \delta\left(E+E^{\prime}-\omega\right)\right]^{2}\left[2 \pi \delta\left(p_{z}+p_{z}{ }^{\prime}-\omega_{z}\right)\right]^{2} \\
& \times \frac{1}{8 E E^{\prime} E_{\mathrm{o}} E_{\mathrm{o}}^{\prime}} I_{s, s^{\prime}}{ }^{2}\left(\frac{\omega^{2} \sin ^{2} \theta}{2 \mathrm{e} B}\right) \\
& \times\left[\sin ^{2} \theta\left(E^{\prime} E+p_{z}{ }^{\prime} p_{z}-r r^{\prime} E_{\mathrm{o}}{ }^{\prime} E_{\mathrm{o}}\right)\left(E_{\mathrm{o}}+r m\right)\right. \\
& \times\left(E_{\mathrm{o}}{ }^{\prime}-r^{\prime} m\right) I_{N^{\prime}-1, N-1}{ }^{2}+\left(E_{\mathrm{o}}{ }^{\prime}+r^{\prime} m\right) \\
& \left.\times\left(E_{\mathrm{o}}-r m\right) I_{N^{\prime}, N^{2}}{ }^{2}\right]+\sin 2 \theta\left(E_{\mathrm{o}}+r m\right) \\
& \times \sqrt{2 \mathrm{e} B N^{\prime}}\left(p_{z} E_{\mathrm{o}}{ }^{\prime}+r r^{\prime} p_{z}{ }^{\prime} E_{\mathrm{o}}\right) I_{N^{\prime}-1, N-1} I_{N^{\prime}, N-1} \\
& +\sin 2 \theta r r^{\prime} \sqrt{2 \mathrm{e} B N}\left(E_{\mathrm{o}}{ }^{\prime}-r^{\prime} m\right)\left(p_{z} E_{\mathrm{o}}{ }^{\prime}+r r^{\prime} p_{z}{ }^{\prime} E_{\mathrm{o}}\right) \\
& \times I_{N^{\prime}-1, N-1} I_{N^{\prime}-1, N}-\sin ^{2} \theta 4 \mathrm{e} B \sqrt{N N^{\prime}}\left(E_{\mathrm{o}}{ }^{\prime} E_{\mathrm{o}}\right. \\
& \left.-r r^{\prime} E^{\prime} E-r r^{\prime} p_{z}{ }^{\prime} p_{z}\right) I_{N^{\prime}-1, N-1} I_{N^{\prime}, N} \\
& +\left(1+\cos ^{2} \theta\right)\left(E^{\prime} E-p_{z}{ }^{\prime} p_{z}+r r^{\prime} E_{\mathrm{o}}{ }^{\prime} E_{\mathrm{o}}\right) \\
& \times\left[\left(E_{\mathrm{o}}{ }^{\prime}+r^{\prime} m\right)\left(E_{\mathrm{o}}+r m\right) I_{N^{\prime}, N-1}{ }^{2}+\left(E_{\mathrm{o}}-r m\right)\right. \\
& \left.\times\left(E_{\mathrm{o}}{ }^{\prime}-r^{\prime} m\right) I_{N^{\prime}-1, N^{2}}{ }^{2}\right]-4 \mathrm{e} B \sqrt{N N^{\prime}} \sin ^{2} \theta \\
& \times\left(r r^{\prime} E^{\prime} E-r r^{\prime} p_{z}{ }^{\prime} p_{z}+E_{\mathrm{o}}{ }^{\prime} E_{\mathrm{O}}\right) I_{N^{\prime}, N-1} I_{N^{\prime}-1, N} \\
& +\sin 2 \theta\left(p_{z} E_{\mathrm{o}}{ }^{\prime}+r r^{\prime} p_{z}{ }^{\prime} E_{\mathrm{o}}\right) I_{N^{\prime}, N} \\
& \times\left[r r^{\prime} \sqrt{2 \mathrm{e} B N}\left(E_{\mathrm{o}}{ }^{\prime}+r^{\prime} m\right) I_{N^{\prime}, N-1}+\left(E_{\mathrm{o}}-r m\right)\right. \\
& \left.\times \sqrt{2 \mathrm{e} B N^{\prime}} I_{N^{\prime}-1, N}\right] \text {. }
\end{aligned}
$$


For the particular case $p_{z}=0$, the probability of this process for unpolarized radiation and a polarized electronpositron pair is:

$$
\begin{aligned}
& \left|S_{\mathrm{fi}}\right|_{p_{z}=0}^{2}=\frac{\mathrm{e}^{2}}{4 \omega V} \frac{1}{L^{2}}\left[2 \pi \delta\left(E_{\mathrm{o}}+E^{\prime}-\omega\right)\right]^{2}\left[2 \pi \delta\left(p_{z}{ }^{\prime}-\omega_{z}\right)\right]^{2} \\
& \times \frac{1}{8 E^{\prime} E_{\mathrm{o}} E_{\mathrm{o}}^{\prime}} I_{s, s^{\prime}}{ }^{2}\left(\frac{\omega^{2} \sin ^{2} \theta}{2 \mathrm{e} B}\right) \\
& \times\left[\operatorname { s i n } ^ { 2 } \theta ( E ^ { \prime } - r r ^ { \prime } E _ { \mathrm { o } } { } ^ { \prime } ) \left[\left(E_{\mathrm{o}}+r m\right)\left(E_{\mathrm{o}}{ }^{\prime}-r^{\prime} m\right)\right.\right. \\
& \times I_{N^{\prime}-1, N-1}{ }^{2}+\left(E_{\mathrm{o}}{ }^{\prime}+r^{\prime} m\right)\left(E_{\mathrm{o}}-r m\right) \\
& \left.\times I_{N^{\prime}, N^{2}}^{2}\right]+\sin 2 \theta\left(E_{\mathrm{o}}+r m\right) \sqrt{2 \mathrm{e} B N^{\prime}} r r^{\prime} p_{z}{ }^{\prime} \\
& \times I_{N^{\prime}-1, N-1} I_{N^{\prime}, N-1}+\sin 2 \theta \sqrt{2 \mathrm{e} B N}\left(E_{\mathrm{o}}{ }^{\prime}\right. \\
& \left.-r^{\prime} m\right) p_{z}{ }^{\prime} I_{N^{\prime}-1, N-1} I_{N^{\prime}-1, N}-\sin ^{2} \theta 4 \mathrm{e} B \\
& \times \sqrt{N N^{\prime}}\left(E_{\mathrm{o}}{ }^{\prime}-r r^{\prime} E^{\prime}\right) I_{N^{\prime}-1, N-1} I_{N^{\prime}, N} \\
& +\left(1+\cos ^{2} \theta\right)\left(E^{\prime}+r r^{\prime} E_{\mathrm{o}}{ }^{\prime}\right)\left[\left(E_{\mathrm{o}}{ }^{\prime}+r^{\prime} m\right)\right. \\
& \times\left(E_{\mathrm{o}}+r m\right) I_{N^{\prime}, N-1}{ }^{2}+\left(E_{\mathrm{o}}-r m\right) \\
& \left.\times\left(E_{\mathrm{o}}{ }^{\prime}-r^{\prime} m\right) I_{N^{\prime}-1, N^{2}}{ }^{2}\right]-4 \mathrm{e} B \sqrt{N N^{\prime}} \sin ^{2} \theta \\
& \times\left(r r^{\prime} E^{\prime}+E_{\mathrm{o}}{ }^{\prime}\right) I_{N^{\prime}, N-1} I_{N^{\prime}-1, N}+\sin 2 \theta \\
& \times \sqrt{2 \mathrm{e} B N}\left(E_{\mathrm{o}}{ }^{\prime}+r^{\prime} m\right) p_{z}{ }^{\prime} I_{N^{\prime}, N-1} I_{N^{\prime}, N} \\
& +\sin 2 \theta\left(E_{\mathrm{o}}-r m\right) \sqrt{2 \mathrm{e} B N^{\prime}} r r^{\prime} p_{z}{ }^{\prime} \\
& \left.\times I_{N^{\prime}-1, N} I_{N^{\prime}, N}\right] \text {. }
\end{aligned}
$$

If we allow any polarization for the electron in the above we obtain:

$$
\begin{aligned}
& \left|S_{\mathrm{fi}}\right|_{p_{z}=0}^{2}=\frac{\mathrm{e}^{2}}{4 \omega V} \frac{1}{L^{2}}\left[2 \pi \delta\left(E_{\mathrm{o}}+E^{\prime}-\omega\right)\right]^{2}\left[2 \pi \delta\left(p_{z}{ }^{\prime}-\omega_{z}\right)\right]^{2} \\
& \times \frac{1}{4 E^{\prime} E_{\mathrm{o}} E_{\mathrm{o}}^{\prime}} I_{s, s^{\prime}}{ }^{2}\left(\frac{\omega^{2} \sin ^{2} \theta}{2 \mathrm{e} B}\right) \frac{\left(1+\delta_{N, 0}\right)}{2} \\
& \times\left[\operatorname { s i n } ^ { 2 } \theta \left[\left(E_{\mathrm{o}}{ }^{\prime}-r^{\prime} m\right)\left(E^{\prime} E_{\mathrm{o}}-r^{\prime} E_{\mathrm{o}}{ }^{\prime} m\right)\right.\right. \\
& \times I_{N^{\prime}-1, N-1}{ }^{2}+\left(E_{\mathrm{o}}{ }^{\prime}+r^{\prime} m\right) \\
& \left.\times\left(E^{\prime} E_{\mathrm{o}}+r^{\prime} E_{\mathrm{o}}{ }^{\prime} m\right) I_{N^{\prime}, N}{ }^{2}\right]
\end{aligned}
$$$$
+\sin 2 \theta \sqrt{2 \mathrm{e} B N^{\prime}} m r^{\prime} p_{z}{ }^{\prime} I_{N^{\prime}-1, N-1} I_{N^{\prime}, N-1}
$$$$
+\sin 2 \theta \sqrt{2 \mathrm{e} B N}\left(E_{\mathrm{o}}{ }^{\prime}-r^{\prime} m\right) p_{z}{ }^{\prime}
$$$$
\times I_{N^{\prime}-1, N-1} I_{N^{\prime}-1, N}
$$$$
-\sin ^{2} \theta 4 \mathrm{e} B \sqrt{N N^{\prime}} E_{\mathrm{o}}{ }^{\prime} I_{N^{\prime}-1, N-1} I_{N^{\prime}, N}
$$$$
+\left(1+\cos ^{2} \theta\right)\left[\left(E_{\mathrm{o}}{ }^{\prime}+r^{\prime} m\right)\left(E^{\prime} E_{\mathrm{o}}+r^{\prime} E_{\mathrm{o}}{ }^{\prime} m\right)\right.
$$$$
\times I_{N^{\prime}, N-1}{ }^{2}+\left(E_{\mathrm{o}}{ }^{\prime}-r^{\prime} m\right)\left(E^{\prime} E_{\mathrm{o}}-r^{\prime} E_{\mathrm{o}}{ }^{\prime} m\right)
$$$$
\left.\times I_{N^{\prime}-1, N}^{2}\right]
$$$$
-4 \mathrm{e} B \sqrt{N N^{\prime}} \sin ^{2} \theta E_{\mathrm{o}}{ }^{\prime} I_{N^{\prime}, N-1} I_{N^{\prime}-1, N}
$$$$
+\sin 2 \theta \sqrt{2 \mathrm{e} B N}\left(E_{\mathrm{o}}{ }^{\prime}+r^{\prime} m\right) p_{z}{ }^{\prime} I_{N^{\prime}, N-1} I_{N^{\prime}, N}
$$$$
\left.-\sin 2 \theta m r^{\prime} p_{z}{ }^{\prime} \sqrt{2 \mathrm{e} B N^{\prime}} I_{N^{\prime}-1, N} I_{N^{\prime}, N}\right] \text {. }
$$

Summing over the polarization of the positron one obtains the probability for pair creation by an unpolarized photon of an electron-positron pair with any polarization:

$$
\begin{aligned}
\left|S_{\mathrm{fi}}\right|_{p_{z}=0}^{2} & =\frac{\mathrm{e}^{2}}{4 \omega V} \frac{1}{L^{2}}\left[2 \pi \delta\left(E_{\mathrm{o}}+E^{\prime}-\omega\right)\right]^{2}\left[2 \pi \delta\left(p_{z}{ }^{\prime}-\omega_{z}\right)\right]^{2} \\
& \times \frac{1}{2 E^{\prime} E_{\mathrm{o}}} I_{s, s^{\prime}}{ }^{2}\left(\frac{\omega^{2} \sin ^{2} \theta}{2 \mathrm{e} B}\right) \frac{\left(1+\delta_{N, 0}\right)\left(1+\delta_{N^{\prime}, 0}\right)}{4}
\end{aligned}
$$

$$
\begin{aligned}
& {\left[\operatorname { s i n } ^ { 2 } \theta \left[\left(E^{\prime} E_{\mathrm{O}}+m^{2}\right)\left[I_{N^{\prime}-1, N-1}{ }^{2}+I_{N^{\prime}, N^{2}}^{2}\right]\right.\right.} \\
& +\sin 2 \theta \sqrt{2 \mathrm{e} B N} p_{z}{ }^{\prime} \\
& \times\left[I_{N^{\prime}-1, N-1} I_{N^{\prime}-1, N}+I_{N^{\prime}, N-1} I_{N^{\prime}, N}\right] \\
& -\sin ^{2} \theta 4 \mathrm{e} B \sqrt{N N^{\prime}} \\
& \left.\times\left[I_{N^{\prime}-1, N-1} I_{N^{\prime}, N}+I_{N^{\prime}, N-1} I_{N^{\prime}-1, N}\right]\right] \\
& +\left(1+\cos ^{2} \theta\right)\left(E^{\prime} E_{\mathrm{O}}+m^{2}\right) \\
& \times\left[I_{N^{\prime}, N-1}{ }^{2}+I_{N^{\prime}-1, N}^{2}\right] .
\end{aligned}
$$

\section{Results}

The attenuation coefficients for photons may be written as:

$$
R=\frac{1}{T} \sum_{N \geq 0} \sum_{N^{\prime} \geq 0} \int \frac{L \mathrm{~d} p_{z}}{2 \pi} \int \frac{L \mathrm{~d} p_{z}{ }^{\prime}}{2 \pi}\left|S_{\mathrm{fi}}\right|^{2} .
$$

In the case of a polarized photon and a polarized electronpositron pair this yields:

$$
R(\omega, \theta, B)=\sum_{N \geq 0} \sum_{N^{\prime} \geq 0} \frac{\alpha}{4 E_{\mathrm{o}} E_{\mathrm{o}}{ }^{\prime} \omega} \frac{m^{2} B^{\prime}}{\left|p_{z} \cdot E^{\prime}-p_{z^{\prime}} \cdot E\right|}\left[N_{\mathrm{p}}\right]
$$

with $\left[N_{\mathrm{p}}\right]$ defined above and $\alpha \simeq 1 / 137.037$ is the finestructure constant. In Eq. (28) both solutions for $p_{z}{ }^{\prime}$ from energy and momentum conservation have been included. So, if $\mathrm{e}^{-}$and $\mathrm{e}^{+}$are found in the same Landau quantum levels $\left(N=N^{\prime}\right)$ we have singularities in the energy: $\omega=$ $2 m\left[1+2 B^{\prime} N\right]^{1 / 2} / \sin \theta$, in this particular case. For $N \neq$ $N^{\prime}$, the expression for $\omega$ is more complicated.

In the case of a polarized photon and an electronpositron pair with any polarization, this yields:

$$
\begin{aligned}
R(\omega, \theta, B)= & \sum_{N \geq 0} \sum_{N^{\prime} \geq 0} \frac{\alpha}{\omega} \frac{m^{2} B^{\prime}}{\left|p_{z} \cdot E^{\prime}-p_{z}{ }^{\prime} \cdot E\right|}\left[N_{\mathrm{p}}\right]_{3} \\
& \times \frac{\left(1+\delta_{N, 0}\right)\left(1+\delta_{N^{\prime}, 0}\right)}{4}
\end{aligned}
$$

with $\left[N_{\mathrm{p}}\right]_{3}$ defined above.

If we take the case $\theta=90^{\circ}$, which implies that $p_{z}=-p_{z}{ }^{\prime}$, any polarization for the pair, and linear photon polarization $\varepsilon^{(1)}$, we obtain:

$$
\begin{aligned}
R_{\|}\left(\omega^{\prime}, \pi / 2, B^{\prime}\right)= & \frac{\alpha}{2 \rho} \sum_{N \geq 0} \sum_{N^{\prime} \geq 0} \frac{\left(1+\delta_{N, 0}\right)\left(1+\delta_{N^{\prime}, 0}\right)}{4\left|p_{z^{\prime}}\right|} \\
& \times\left[( E E ^ { \prime } - p _ { z } { } ^ { 2 } + m ^ { 2 } ) \left(I_{N^{\prime}-1, N-1}{ }^{2}(\rho)\right.\right. \\
& \left.+I_{N^{\prime}, N}{ }^{2}(\rho)\right)-4 m^{2} B^{\prime} \sqrt{N^{\prime}} \\
& \left.\times I_{N^{\prime}-1, N-1}(\rho) I_{N^{\prime}, N}(\rho)\right]
\end{aligned}
$$

with $\omega^{\prime}=\omega /(2 m)$ and $\rho=2 \omega^{\prime 2} / B^{\prime}$. For linear polarization $\varepsilon^{(2)}$, we obtain:

$$
\begin{aligned}
R_{\perp}\left(\omega^{\prime}, \pi / 2, B^{\prime}\right)= & \frac{\alpha}{2 \rho} \sum_{N \geq 0} \sum_{N^{\prime} \geq 0} \frac{\left(1+\delta_{N, 0}\right)\left(1+\delta_{N^{\prime}, 0}\right)}{4\left|p_{z^{\prime}}\right|} \\
& \times\left[( E E ^ { \prime } + p _ { z } { } ^ { 2 } + m ^ { 2 } ) \left(I_{N^{\prime}, N-1}{ }^{2}(\rho)\right.\right. \\
& \left.+I_{N^{\prime}-1, N}{ }^{2}(\rho)\right)-4 m^{2} B^{\prime} \sqrt{N N^{\prime}} \\
& \left.\times I_{N^{\prime}, N-1}(\rho) I_{N^{\prime}-1, N}(\rho)\right] .
\end{aligned}
$$


This differs from the result of Daugherty \& Harding (1983): they omit the factor $\frac{\left(1+\delta_{N, 0}\right)\left(1+\delta_{N^{\prime}, 0}\right)}{4}$. This factor was also obtained by Frangodimitraki-Georgiadou (1991). The relation between the function $M_{N, N^{\prime}}(\rho)$ of Daugherty \& Harding (1983) and the Laguerre function is:

$M_{N, N^{\prime}}(\rho)=i^{-\left(N+N^{\prime}\right)} I_{N, N^{\prime}}(\rho)$.

The attenuation coefficients have a simple form if we take the case of any polarization pair formed in: fundamental Landau states $\left(N=N^{\prime}=0\right)$; or fundamental plus first excited Landau states $\left(N=0, N^{\prime}=1\right.$ or $\left.N=1, N^{\prime}=0\right)$. The result is:

$$
\begin{aligned}
R_{\|}\left(\omega^{\prime}, \pi / 2, B^{\prime}\right)_{0,0} & =\frac{\alpha m^{2}}{\rho\left|p_{0,0^{\prime} \mid}\right|} \mathrm{e}^{-\rho} \\
& =\frac{\alpha}{\rho \lambda_{c}{ }^{2}\left|p_{0,0^{\prime}}\right|} \mathrm{e}^{-\rho}
\end{aligned}
$$

with $p_{0,0^{\prime}}=m\left[\frac{\omega^{2}}{4 m^{2}}-1\right]^{1 / 2}$ and $A_{\mathrm{c}}=\lambda_{\mathrm{c}} /(2 \pi) \simeq 3.8616 \times$ $10^{-11} \mathrm{~cm}$ is the Compton wavelength of the electron. In the above expression, momentum is expressed in energy units (e.g. $\mathrm{MeV}$ ), as is $\mathrm{m}$, so the first line of (33) gives $R$ in energy units (e.g. $1 \mathrm{MeV}$ is equivalent to $5.06777 \times 10^{10} \mathrm{~cm}^{-1}$ in units with $c=\hbar=1$ ). If we express momentum in terms of units of electron rest mass, $\mathrm{m}$, the expression is:

$R_{\|}\left(\omega^{\prime}, \pi / 2, B^{\prime}\right)_{0,0}=\frac{\alpha}{\rho \lambda_{c}\left|p_{0,0^{\prime}}\right|} \mathrm{e}^{-\rho}$

with $p_{0,0^{\prime}}=\left[\frac{\omega^{2}}{4 m^{2}}-1\right]^{1 / 2}$. This same expression was given by Harding et al. (1997).

For the pair formed in the fundamental state $(N=$ $\left.N^{\prime}=0\right), R_{\perp, 0,0}=0$, so when the electric field vector of the radiation is perpendicular to $\boldsymbol{B}$, the fundamental Landau state does not contribute to attenuation of this type of radiation. Thus pairs formed with $N=N^{\prime}=$ 0 are created only by radiation with linear polarization $\varepsilon^{\hat{(1)}}$ or with circular polarization $\hat{\varepsilon}_{( \pm)}$with $R_{\|, 0,0, \varepsilon^{(1)}}=$ $2 R_{\|, 0,0, \varepsilon_{( \pm)}}$.

In the case of a pair with any polarization, $\theta=\pi / 2$, and $N=0, N^{\prime}=1$ or $N=1, N^{\prime}=0$ one finds:

$$
\begin{aligned}
R_{\|}\left(\omega^{\prime}, \pi / 2, B^{\prime}\right)_{0,1}= & R_{\|}\left(\omega^{\prime}, \pi / 2, B^{\prime}\right)_{1,0}=\frac{\alpha}{4\left|p_{z^{\prime}}\right|_{0,1}} \\
& \times\left(E^{\circ} E_{1}^{\prime}-p_{z_{0,1}^{\prime}}{ }^{2}+m^{2}\right) \mathrm{e}^{-\rho}
\end{aligned}
$$

for $\quad E^{\mathrm{o}}, E_{1}^{\prime}, p_{z_{0,1}^{\prime}}^{\prime} \quad$ in energy units, with $\left|p_{z^{\prime}}\right|_{0,1}=m \sqrt{{\omega^{\prime}}^{2}-1-B^{\prime}+\frac{B^{\prime 2}}{4 \omega^{\prime 2}}} \quad\left(\right.$ or $\quad\left|p_{z}\right|_{0,1}=$ $\sqrt{{\omega^{\prime}}^{2}-1-B^{\prime}+\frac{B^{\prime 2}}{4 \omega^{\prime 2}}}$ in units of $\left.m\right)$. The energy for the electron is $E^{\mathrm{o}}=m \sqrt{{\omega^{\prime}}^{2}-B^{\prime}+\frac{B^{\prime 2}}{4 \omega^{\prime 2}}}$ in energy units (or $E^{\mathrm{o}}=\sqrt{{\omega^{\prime}}^{2}-B^{\prime}+\frac{B^{\prime 2}}{4 \omega^{\prime 2}}}$ in units of $m$ ). The energy of the positron is $E_{1}{ }^{\prime}=m \sqrt{\omega^{\prime 2}+B^{\prime}+\frac{B^{\prime 2}}{4 \omega^{\prime 2}}}$ in energy units (or $E_{1}{ }^{\prime}=\sqrt{{\omega^{\prime}}^{2}+B^{\prime}+\frac{B^{\prime 2}}{4 \omega^{\prime 2}}}$ in units of $m$ ). If we sum the rates for $N=0, N^{\prime}=1$ and $N=1, N^{\prime}=0$ we obtain:

$$
\begin{array}{r}
R_{\|}\left(\omega^{\prime}, \pi / 2, B^{\prime}\right)_{0,1}+R_{\|}\left(\omega^{\prime}, \pi / 2, B^{\prime}\right)_{1,0}= \\
\frac{\alpha}{2\left|p_{z}\right|_{0,1}}\left(E^{\circ} E_{1}^{\prime}-\left|p_{z}{ }^{\prime}\right|_{0,1}{ }^{2}+m^{2}\right) \mathrm{e}^{-\rho}
\end{array}
$$

for energy and momentum expressed in energy units. For energy and momentum expressed in units of $m$, replace $m$ by 1 and put a factor of $A_{c}$ in the denominator. For linear polarization $\varepsilon^{\hat{(2)}}$ one has:

$$
\begin{aligned}
R_{\perp}\left(\omega^{\prime}, \pi / 2, B^{\prime}\right)_{0,1}= & R_{\perp}\left(\omega^{\prime}, \pi / 2, B^{\prime}\right)_{1,0} \\
= & \frac{\alpha}{4 \rho\left|p_{z}{ }^{\prime}\right|_{0,1}} \\
& \times\left(E^{\circ} E_{1}^{\prime}+\left|p_{z}{ }^{\prime}\right|_{0,1}{ }^{2}+m^{2}\right) \mathrm{e}^{-\rho}
\end{aligned}
$$

and

$$
\begin{array}{r}
R_{\perp}\left(\omega^{\prime}, \pi / 2, B^{\prime}\right)_{0,1}+R_{\perp}\left(\omega^{\prime}, \pi / 2, B^{\prime}\right)_{1,0}= \\
\frac{\alpha}{2 \rho\left|p_{z^{\prime}}\right|_{0,1}}\left(E^{\circ} E_{1}^{\prime}+\left|p_{z}{ }^{\prime}\right|_{0,1}{ }^{2}+m^{2}\right) \mathrm{e}^{-\rho} .
\end{array}
$$

Both of the above are for $E^{\circ}, E_{1}^{\prime}, p_{z_{0,1}^{\prime}}$ in energy units.

In the equation for the attenuation coefficient $R$, the sum over $N^{\prime}$ has a maximum permitted value. Applying the energy and momentum conservation laws, we find the parallel component of momentum of the positron depends on $N, N^{\prime}, B^{\prime}, \omega$ and $\theta$. Writing $x=\omega \sin \theta$, one obtains:

$p_{z}{ }^{\prime}=\frac{-\cos \theta\left[x^{2}-2 m^{2} B^{\prime}\left(N-N^{\prime}\right)\right] \pm \sqrt{\left[x^{2}-2 m^{2} B^{\prime}\left(N-N^{\prime}\right)\right]^{2}-4 m^{2} x^{2}\left(1+2 B^{\prime} N^{\prime}\right)}}{-2 x \sin \theta}$

For $N=N^{\prime}=0$ in Eq. (39), since the argument of the square root must be 0 or greater so $p_{z}{ }^{\prime}$ is real, one has $\omega_{\min }=2 \mathrm{~m} / \sin \theta$. When $\omega$ is at its minimum value, the energy of the photon divides equally between $\mathrm{e}^{-}$and $\mathrm{e}^{+}$, and also $\mathrm{e}^{-}$and $\mathrm{e}^{+}$each receive half of the momentum $\omega_{z}$ (i.e. $\left.p_{z}=p_{z}{ }^{\prime}=(\omega \cos \theta) / 2\right)$. The component of momentum of the photon perpendicular to the magnetic field $\left(\omega_{\perp}=\omega \sin \theta\right)$ is absorbed by the field.

For the particular case of $\theta=\pi / 2$ one finds:

$$
p_{z}{ }^{\prime}= \pm m \sqrt{{\omega^{\prime}}^{2}-1-B^{\prime}\left(N+N^{\prime}\right)+\frac{B^{\prime 2}\left(N-N^{\prime}\right)^{2}}{4{\omega^{\prime}}^{2}}}
$$

which is identical with the result obtained by Daugherty \& Harding (1983). Since the expression inside the root must be $\geq 0$, one has for $\theta=\pi / 2$ :

$N_{\text {max }}^{\prime}=\frac{2 m^{2} B^{\prime} N+\omega^{2}-2 \omega m \sqrt{1+2 B^{\prime} N}}{2 m^{2} B^{\prime}}$.

In practice the allowed values of $N^{\prime}$ are the integers less than or equal to the value of $N^{\prime}$ max.

For the general case of $\theta$ we find:

$$
\begin{aligned}
N^{\prime}{ }_{\max } & =\frac{2 m^{2} B^{\prime} N+x^{2}}{2 m^{2} B^{\prime}} \\
& -\frac{\sqrt{\left(2 m^{2} B^{\prime} N+x^{2}\right)^{2}-\left(x^{4}-4 m^{2} B^{\prime} N x^{2}+4 m^{4} B^{\prime 2} N^{2}-4 m^{2} x^{2}\right)}}{2 m^{2} B^{\prime}} .
\end{aligned}
$$


We give an improved approximation to that in Daugherty \& Harding (1983) for the number of allowed states as a function of $\omega^{\prime}$ and $B^{\prime}$ for $\theta=90^{\circ}$. We fitted the following analytic expression to the number of states for $B=B_{\mathrm{cr}}$ to $B=10 B_{\text {cr }}$ and for $\omega=2 \mathrm{MeV}$ to $\omega=20 \mathrm{MeV}$.

$N_{\text {states }} \simeq a_{1} \omega^{\prime}\left(\omega^{\prime}+a_{2}\right)\left(\omega^{\prime}+a_{3}\right)\left(\omega^{\prime}+a_{4}\right) / B^{\prime 2}$.

The best-fit values of the constants are:

$a_{1}=0.59 \quad a_{2}=2.8 \quad a_{3}=0.12 \quad a_{4}=0.05$.

In the limit $\omega^{\prime}>>1$ this reduces to:

$N_{\text {states }} \sim 0.59 \omega^{\prime 4} / B^{\prime 2}$.

The number of available states decreases as the angle $\theta$ gets further away from $90^{\circ}$. For example the numbers of states for angles between $\theta=60^{\circ}$ and $\theta=120^{\circ}$, in steps of $\delta \theta=0.1^{\circ}$ was calculated. We find that the mean number of states (averaged over all angles) as a function of $\omega^{\prime}$ and $B^{\prime}$, in the range $B=2 B_{\text {cr }}$ to $B=10 B_{\text {cr }}$ and $\omega=2 \mathrm{MeV}$ to $\omega=20 \mathrm{MeV}$ is fit by the following expression:

$\left.N_{\text {states }} \simeq 0.69 \omega^{\prime}\left(\omega^{\prime}-0.20\right)\left[\left(\omega^{\prime}-4.16\right)^{2}+6.0 \omega^{\prime}\right)\right] / B^{\prime 2}$.

For example, for $B=B_{\text {cr }}$ and $\omega=6 \mathrm{MeV}$, the expression approximating the number of states for $\theta=90^{\circ}$ gives 1065 , whereas the average number of states for angles in the range $\theta=60^{\circ}$ to $\theta=120^{\circ}$ is 876 .

Figure 1a explicitly shows the dependence of $N_{\text {states }}$ (calculated exactly) on $\omega$ for the case $B=B_{\text {cr }}$ and $\theta=90^{\circ}$. Figure $1 \mathrm{~b}$ shows the dependence of $N_{\text {states }}$ (calculated exactly) on $\theta$ (with $\theta=0$ is the horizontal axis) for the case $B=B_{\text {cr }}$ and four values of $\omega$. This clearly shows the decrease of number of states as $\theta$ gets further away from $90^{\circ}$.

Figure 2 illustrates the division of the energy of the photon between the electron and positron as a function of $\theta$. The electron energy divided by $\omega$ is plotted for the case of $\omega=50 \mathrm{MeV}, B=0.01 B_{\text {cr }}$ and $N=N^{\prime}=0$. The two curves are for the two cases of positron momentum from Eq. (39): the plus sign gives the solid curve and the minus sign gives the dashed line. The positron energy is given by the dashed line for the case of the plus sign and by the solid line for the case of the minus sign. For $\theta=90^{\circ}$ electron and positron each receive half the photon energy. As $\theta$ gets further away from $90^{\circ}$, the energy is more unequally distributed.

In Fig. 3 is illustrated the attenuation coefficient, $R$, calculated for various cases of electron and positron spins and photon polarizations and with the photon propagation angle fixed at $\theta=90^{\circ}$. Figure 3 a shows $R$ for $B=B_{\text {cr }}$ and unpolarized radiation for pair production for all electron spin $(r)$ and positron spin $\left(r^{\prime}\right)$ cases. The $r=-1$, $r^{\prime}=-1$ and $r=+1, r^{\prime}=+1$ attenuation coefficients are identical. This shows that the $r=-1, r^{\prime}=+1$ case is most probable and the $r=+1, r^{\prime}=-1$ is least probable. Figure $3 \mathrm{~b}$ shows $R$ vs. $\omega$ for various photon polarizations and for unpolarized radiation, for the case that $\mathrm{e}^{-}, \mathrm{e}^{+}$ spins are $r=-1, r^{\prime}=+1$. Note that $R$ for the $\epsilon(2)$ case

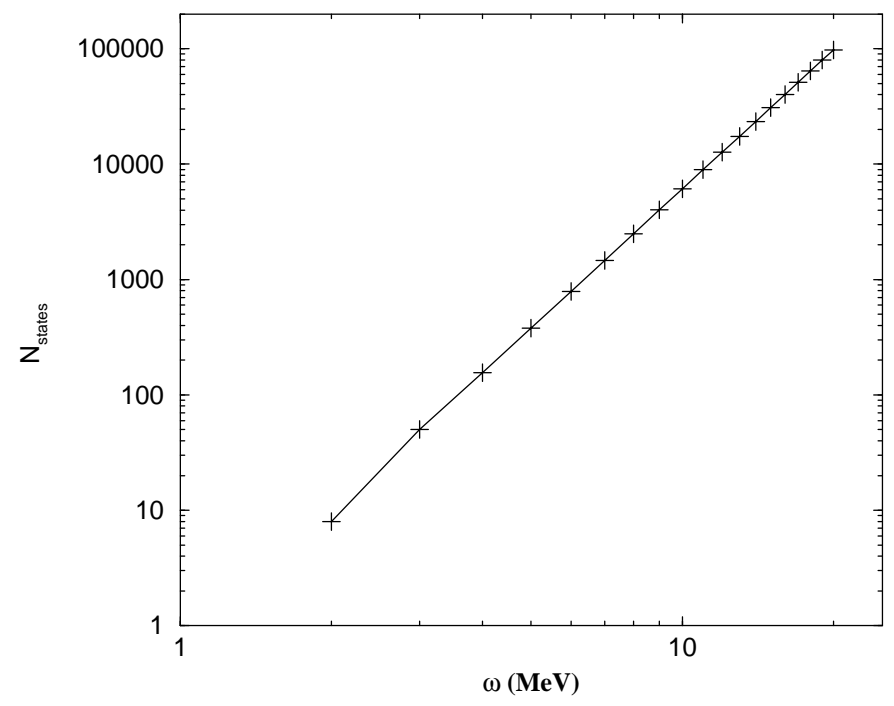

(a)

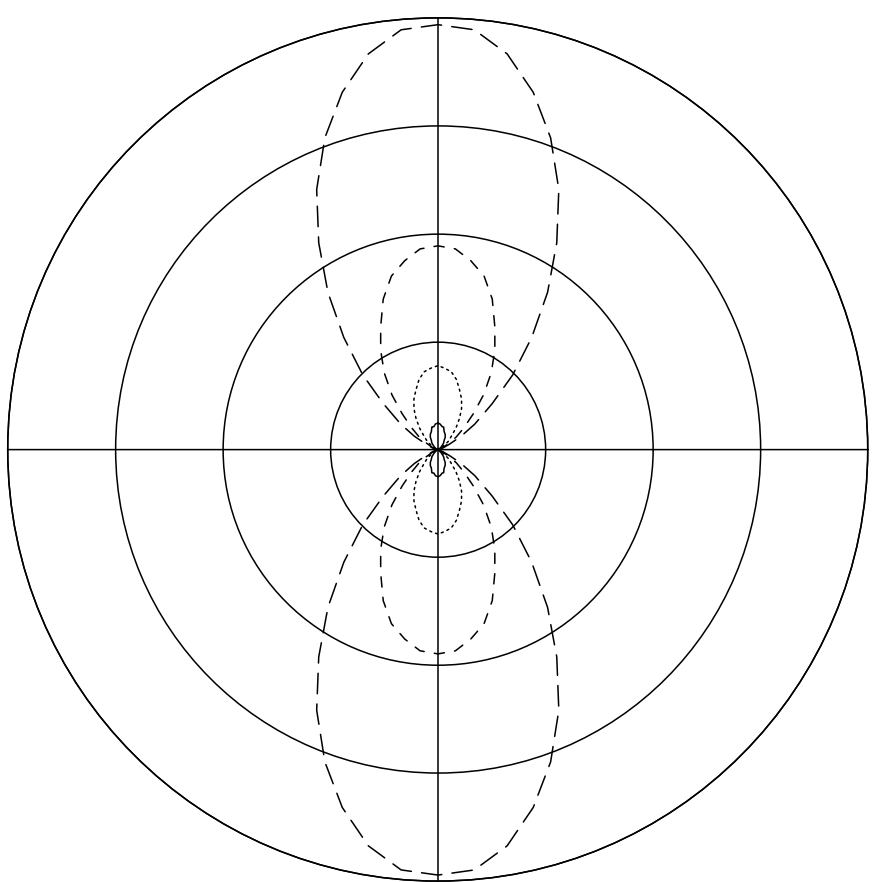

(b)

Fig. 1. a) The number of allowed state as a function of $\omega$ for the case $B=B_{\text {cr }}$ and $\theta=90^{\circ}$. b) The number of allowed state as a function of $\theta$ (with $\theta=0$ is the horizontal axis) for the case $B=B_{\text {cr }}$ and: $\omega=3 \mathrm{MeV}$ (solid line); $\omega=4 \mathrm{MeV}$ (dotted line); $\omega=5 \mathrm{MeV}$ (dashed line); $\omega=6 \mathrm{MeV}$ (long dashed line). The radial circles are at values of 200, 400, 600 and 800 .

has been multiplied by 100 to show it on the same graph as the other cases. This illustrates that the attenuation coefficient for $\epsilon(1)$ radiation is much larger than for $\epsilon(2)$ radiation.

Figure 4 illustrates how $R$ depends on magnetic field and on photon propagation angle. Here we take the radiation is unpolarized and $\mathrm{e}^{-}, \mathrm{e}^{+}$spins are $r=-1, r^{\prime}=+1$. Figure 4 a shows $R$ vs. $\omega$ for five different magnetic field 


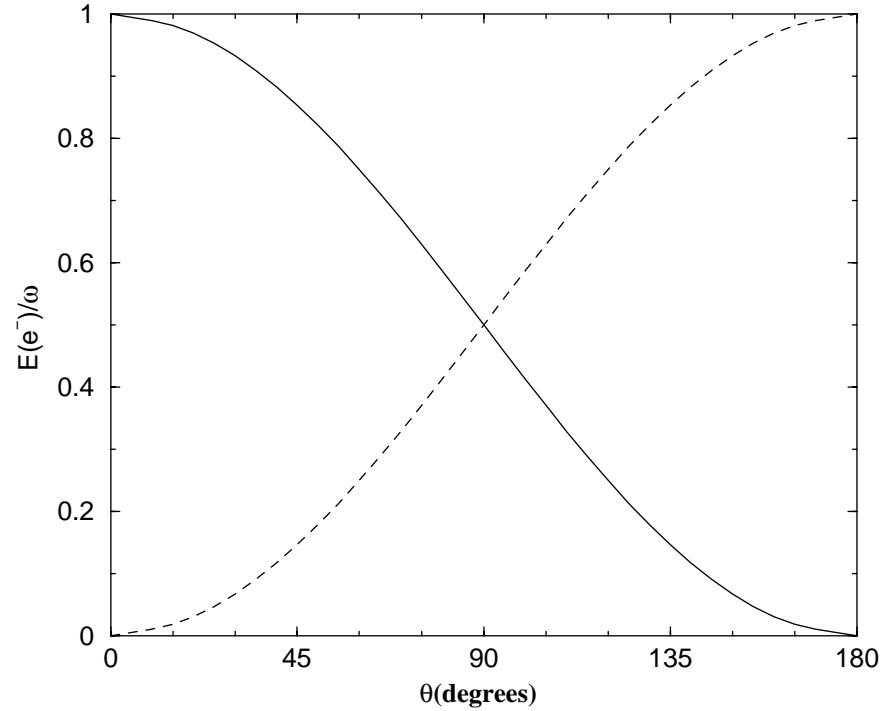

Fig. 2. The ratio of electron energy $\left(E\left(\mathrm{e}^{-}\right)\right)$to photon energy $(\omega)$ as a function of angle $(\theta)$ of the photon to the magnetic field, for the case $N=N^{\prime}=0, B=0.01 B_{\text {cr }}$ and $\omega=50 \mathrm{MeV}$. The solid (dashed) line is for the case of the plus (minus) sign in Eq. (39).

strengths, with $R$ on a logarithmic scale to fit the curves on the same plot. One sees that $R$ increases significantly with increasing $B$ and the spacing between the peaks in $R$ also increases with $B$. Figure $4 \mathrm{~b}$ shows the dependence on $\theta$ for $B=B_{\mathrm{cr}}$. The minimum energy for $R$ to be nonzero obeys $\omega_{\min }=2 m c^{2} / \sin \theta$, but the spectrum also changes due to the dependence of the kinematics on $\theta$.

The last case chosen here takes a photon distribution function to calculate a total pair production rate. This is purely for illustration, as it is a difficult problem (and beyond the scope of this work) to self-consistently determine simultaneously the anisotropic polarized photon distribution and the polarized pair distribution in a strong magnetic field. We take a Wein spectrum for unpolarized photons, with distribution function $f(E)=E^{2} \exp (-E / k T)$, a propagation direction of $90^{\circ}$ to the magnetic field, and include all spin orientations of electron and positron. Figure 5 shows the resulting pair creation rate as a function of temperature for the cases $B=4 B_{\mathrm{cr}}, 2 B_{\mathrm{cr}}, B_{\mathrm{cr}}$, $0.4 B_{\mathrm{cr}}$, and $0.2 B_{\mathrm{cr}}$. The pair creation per photon rate increases rapidly at low temperature (below $0.1 \mathrm{kT} / \mathrm{mc}^{2}$ ), as expected due the sensitivity of the number of photons above threshold to $T$ for $k T / m c^{2}<<1$. The rate also increases rapidly with magnetic field strength. We have also done a calculation including integration over photon propagation angle for $B=10^{13}$ Gauss, with unpolarized photons and summing over electron and positron spins. This case is also plotted in Fig 5. Our results agree with the results in Burns \& Harding (1984) above $0.9 \mathrm{keV}$, but at lower temperatures we obtain a significantly larger rate. Since we use the exact expression for the decay rate, rather than the asymptotic expression valid at high photon energy, this is not surprising. Our results are the correct

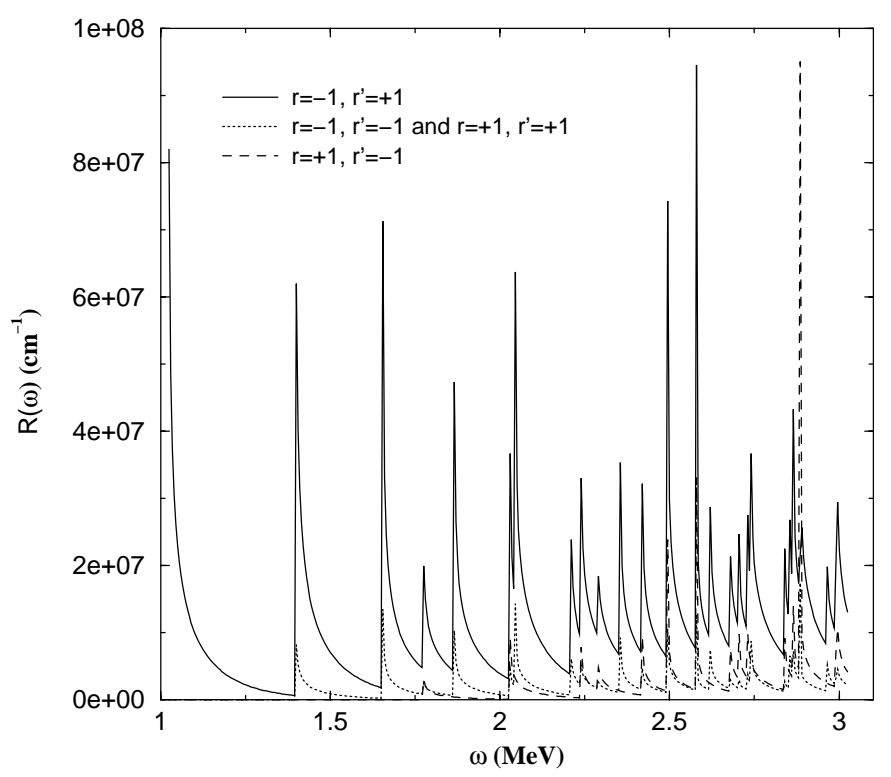

(a)

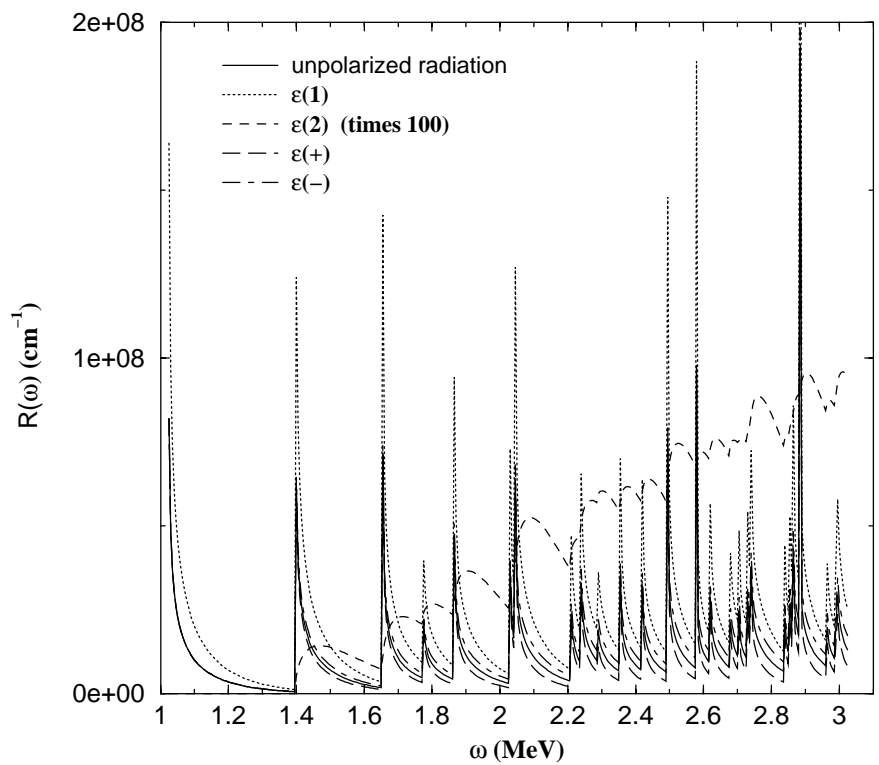

(b)

Fig. 3. The attenuation coefficient for pair production, $R$, as a function of photon energy, $\omega$, for various photon and electronpositron polarizations, with $\theta=\pi / 2$ : a) for different cases of electron spin, $r$, and positron spin, $r^{\prime}$ (with $B=B_{\mathrm{cr}}$ and for unpolarized radiation); $\mathbf{b}$ ) for various photon polarizations (with $B=B_{\text {cr }}$, electron spin $r=-1$ and positron spin $r^{\prime}=+1$.

ones at low temperature, and at high temperature, above $\sim 0.9 \mathrm{keV}$ both calculations agree. At temperatures much above $1 \mathrm{keV}$, the exact expressions for the rate become increasingly difficult to evaluate due to the huge number of final states that must be included in the calculation. 


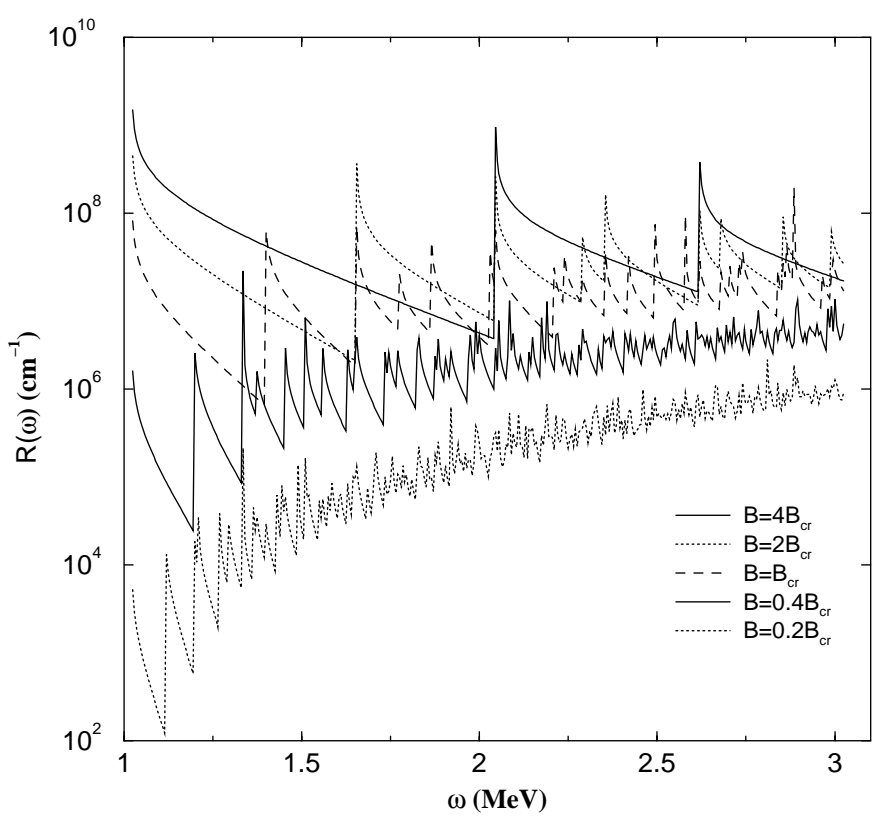

(a)

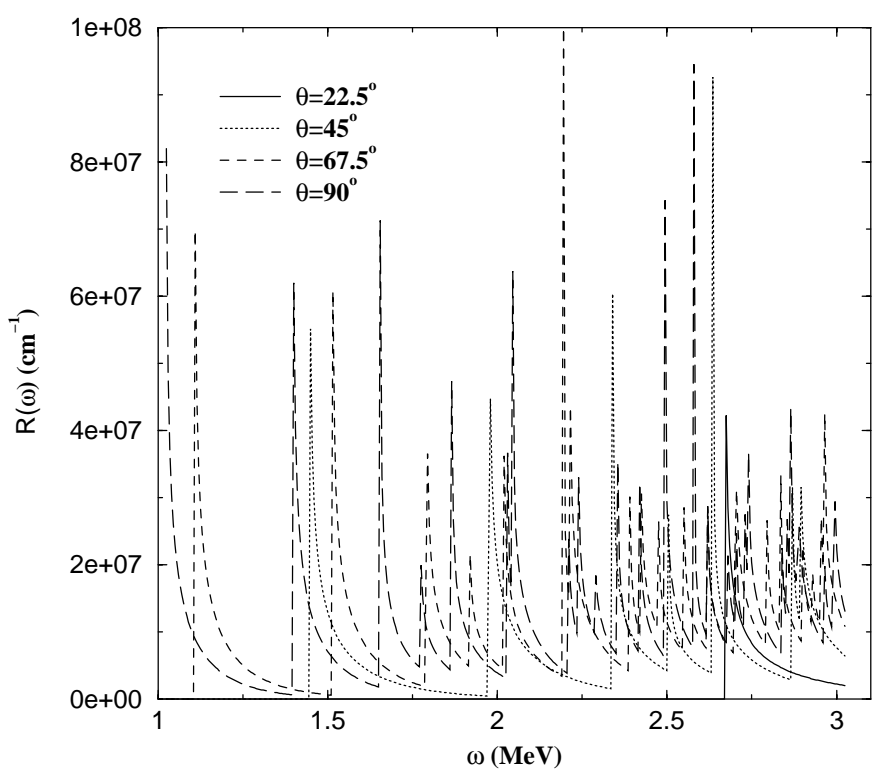

(b)

Fig. 4. The attenuation coefficient for pair production, $R$, as a function of photon energy, $\omega$ (with photon polarization $\epsilon(1)$, electron spin $r=-1$ and positron spin $r^{\prime}=+1$ ), a) for magnetic field values of $B=0.2 B_{\mathrm{cr}}, 0.4 B_{\mathrm{cr}}, B_{\mathrm{cr}}, 2 B_{\mathrm{cr}}$, and $4 B_{\mathrm{cr}}$ and with $\theta=\pi / 2$; b) for different $\theta$ with $B=B_{\mathrm{cr}}$.

\section{Conclusions}

We have derived the probability and attenuation coefficients for pair creation of polarized electrons and positrons by photons in a strong magnetic field. The positron and electron can occupy arbitrary Landau levels and the attenuation coefficient is evaluated for any direction and polarization of the photon. When we take $\theta=90^{\circ}$ and sum

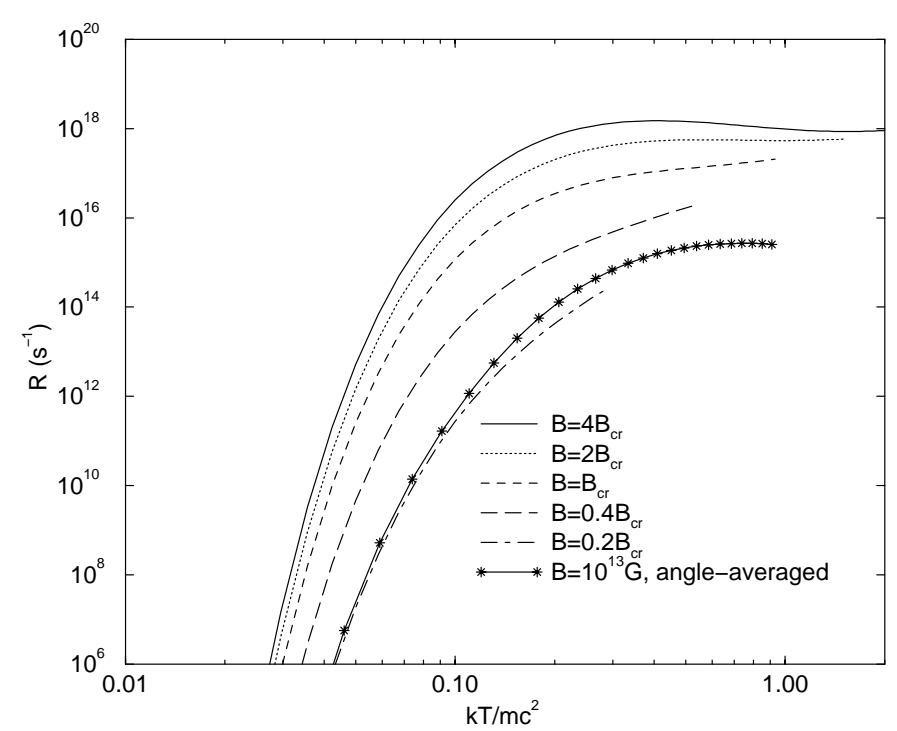

Fig. 5. The attenuation rate for pair production, $R$, for a Wein spectrum of photons, as a function of temperature: for a fixed photon propagation angle $\left(90^{\circ}\right)$, given for magnetic field values of $B=4 B_{\mathrm{cr}}, 2 B_{\mathrm{cr}}, B_{\mathrm{cr}}, 0.4 B_{\mathrm{cr}}$, and $0.2 B_{\mathrm{cr}}$; integrated over solid angle, given for $B=10^{13}$ Gauss.

over polarization of the spins of the $\mathrm{e}^{+}-\mathrm{e}^{-}$pair, we obtain results which differ from the expressions for the attenuation coefficient obtained by Daugherty \& Harding (1983), by the factor $\left(1+\delta_{N, 0}\right)\left(1+\delta_{N^{\prime}, 0}\right) / 4$, in agreement with Frangodimitraki-Georgiadou (1991).

The advance of the current work over previous work is that the attenuation coefficient is now evaluated for all cases of photon polarization and of electron and positron spin states. This was made possible by using the proper spin eigenfunctions for $\mathrm{e}^{-}$and $\mathrm{e}^{+}$in a strong magnetic field, as defined by Sokolov \& Ternov (1983). Polarized pair annihilation rates have presented in Semionova \& Leahy (2000), and polarized photon emission rates in Semionova \& Leahy (1999). With the polarized pair creation attentuation coefficients presented here, one is one step closer to being able to properly study the polarizedphoton/ spin-polarized- $\mathrm{e}^{-}-\mathrm{e}^{+}$radiation transfer problem in strong magnetic fields. This problem requires polarized rates for all of the relevant processes involving photon emission by electrons and positrons as well as pair creation by photons.

Acknowledgements. The authors thank the Natural Sciences and Engineering Research Council of Canada and the Ministry and Science and Technology of Costa Rica and Conicit for support.

\section{Appendix}

We include here some details related to the evaluation of the probability for the first order quantum-electrodynamic process of creation of pairs. We use the wave functions for 
$\mathrm{e}^{-}$and $\mathrm{e}^{+}$defined by Sokolov \& Ternov (1983). For the electron one has:

$$
\begin{aligned}
& \Psi_{\mathrm{e}^{-}}(\boldsymbol{r}, t)_{r= \pm 1}=\mathrm{e}^{-i E t} \mathrm{e}^{i p_{z} z} \sqrt{\frac{\mathrm{e} B}{2 \pi L}} \frac{1}{2 \sqrt{2 E E_{\mathrm{o}}}} \\
& \times\left(\begin{array}{c}
\sqrt{E_{\mathrm{o}} \pm m}\left(\sqrt{E+p_{z}} \pm \sqrt{E-p_{z}}\right) I_{N-1, S}(\rho) \mathrm{e}^{i(l-1) \phi} \\
i \sqrt{E_{\mathrm{o}} \mp m}\left(\sqrt{E-p_{z}} \mp \sqrt{E+p_{z}}\right) I_{N, S}(\rho) \mathrm{e}^{i l \phi} \\
\sqrt{E_{\mathrm{o}} \pm m}\left(\sqrt{E+p_{z}} \mp \sqrt{E-p_{z}}\right) I_{N-1, S}(\rho) \mathrm{e}^{i(l-1) \phi} \\
i \sqrt{E_{\mathrm{o}} \mp m}\left(\sqrt{E-p_{z}} \pm \sqrt{E+p_{z}}\right) I_{N, S}(\rho) \mathrm{e}^{i l \phi}
\end{array}\right),
\end{aligned}
$$

and for the positron one has:

$$
\begin{aligned}
& \Psi_{\mathrm{e}^{+}}(\boldsymbol{r}, t)_{r^{\prime}= \pm 1}=\mathrm{e}^{i E^{\prime} t} \mathrm{e}^{-i p_{z^{\prime}} z} \sqrt{\frac{e B}{2 \pi L}} \frac{1}{2 \sqrt{2 E^{\prime} E_{\mathrm{O}}}} \\
& \times\left(\begin{array}{c}
\sqrt{E_{\mathrm{O}}^{\prime} \mp m}\left(\sqrt{E^{\prime}-p_{z^{\prime}}} \pm \sqrt{\left.E^{\prime}+p_{z^{\prime}}\right) I_{N^{\prime}-1, S^{\prime}}(\rho) \mathrm{e}^{i\left(l^{\prime}-1\right) \phi}}\right. \\
-i \sqrt{E_{\mathrm{O}^{\prime}} \pm m}\left(\sqrt{E^{\prime}+p_{z^{\prime}}} \mp \sqrt{E^{\prime}-p_{z^{\prime}}}\right) I_{N^{\prime}, S^{\prime}}(\rho) \mathrm{e}^{i l^{\prime} \phi} \\
\sqrt{E_{\mathrm{o}}^{\prime} \mp m}\left(\sqrt{E^{\prime}-p_{z^{\prime}}} \mp \sqrt{E^{\prime}+p_{z^{\prime}}}\right) I_{N^{\prime}-1, S^{\prime}}(\rho) \mathrm{e}^{i\left(l^{\prime}-1\right) \phi} \\
-i \sqrt{E_{\mathrm{o}}^{\prime} \pm m}\left(\sqrt{E^{\prime}+p_{z^{\prime}}} \pm \sqrt{E^{\prime}-p_{z^{\prime}}}\right) I_{N^{\prime}, S^{\prime}}(\rho) \mathrm{e}^{i l^{\prime} \phi}
\end{array}\right) .
\end{aligned}
$$

The integration about $p_{z}$ is:

$\int_{0}^{\infty} \mathrm{d} p_{z} \delta\left(E+E^{\prime}-\omega\right) \delta\left(p_{z}+p_{z}{ }^{\prime}-\omega_{z}\right) g\left(p_{z}\right)$

where we apply the property of the $\delta$-function:

$\delta(f(x))=\sum_{\alpha} \frac{\delta\left(x-x_{\alpha}\right)}{|\partial f / \partial x|_{x=x_{\alpha}}}$.

When we take:

$$
\begin{aligned}
f\left(p_{z}\right)= & E+E^{\prime}-\omega \\
= & \sqrt{m^{2}+p_{z}^{2}+2 \mathrm{e} B N} \\
& +\sqrt{m^{2}+\left(\omega \cos \theta-p_{z}\right)^{2}+2 \mathrm{e} B N^{\prime}}-\omega
\end{aligned}
$$

we obtain:

$\delta\left(E+E^{\prime}-\omega\right)=\sum_{\alpha} \frac{\delta\left(p_{z}-p_{z_{\alpha}}\right)}{\left|\frac{p_{z} E^{\prime}-p_{z^{\prime}} E}{E E^{\prime}}\right|}$

and the integration about $p_{z}$ above gives:

$$
\frac{E E^{\prime}}{\left|p_{z \alpha} E^{\prime}-p_{z}^{\prime} E\right|} g\left(p_{z \alpha}\right) \text {. }
$$

Four integrals required to evaluate $S_{\text {fi }}$ are:

$$
\begin{aligned}
I_{1}= & \int_{0}^{\infty} \mathrm{d} \rho \int_{0}^{2 \pi} \mathrm{d} \phi \mathrm{e}^{i \omega r \sin \theta \cos (\varphi-\phi)} \\
& \times I_{N-1, S}(\rho) I_{N^{\prime}, S^{\prime}}(\rho) \mathrm{e}^{-i\left(l-1-l^{\prime}\right) \phi} \\
= & 2 \pi i^{l^{\prime}-l+1} \mathrm{e}^{i\left(l^{\prime}-l+1\right) \varphi}(-1)^{S+S^{\prime}} \\
& \times I_{S, S^{\prime}}\left(\frac{\omega^{2} \sin ^{2} \theta}{2 \mathrm{e} B}\right) I_{N^{\prime}, N-1}\left(\frac{\omega^{2} \sin ^{2} \theta}{2 \mathrm{e} B}\right) \\
I_{2}= & \int_{0}^{\infty} \mathrm{d} \rho \int_{0}^{2 \pi} \mathrm{d} \phi \mathrm{e}^{i \omega r} \sin \theta \cos (\varphi-\phi) \\
& \times I_{N, S}(\rho) I_{N^{\prime}-1, S^{\prime}}(\rho) \mathrm{e}^{-i\left(l+1-l^{\prime}\right) \phi} \\
= & 2 \pi i^{l^{\prime}-l-1} \mathrm{e}^{i\left(l^{\prime}-l-1\right) \varphi}(-1)^{S+S^{\prime}} \\
& \times I_{S, S^{\prime}}\left(\frac{\omega^{2} \sin ^{2} \theta}{2 \mathrm{e} B}\right) I_{N^{\prime}-1, N}\left(\frac{\omega^{2} \sin ^{2} \theta}{2 \mathrm{e} B}\right) \\
I_{3}= & \int_{0}^{\infty} \mathrm{d} \rho \int_{0}^{2 \pi} \mathrm{d} \phi \mathrm{e}^{i \omega r \sin \theta \cos (\varphi-\phi)} \\
& \times I_{N-1, S}(\rho) I_{N^{\prime}-1, S^{\prime}}(\rho) \mathrm{e}^{-i\left(l-l^{\prime}\right) \phi} \\
= & 2 \pi i^{l^{\prime}-l} \mathrm{e}^{i\left(l^{\prime}-l\right) \varphi}(-1)^{S+S^{\prime}} \\
& \times I_{S, S^{\prime}}\left(\frac{\omega^{2} \sin ^{2} \theta}{2 \mathrm{e} B}\right) I_{N^{\prime}-1, N-1}\left(\frac{\omega^{2} \sin ^{2} \theta}{2 \mathrm{e} B}\right)
\end{aligned}
$$

$$
\begin{aligned}
I_{4}= & \int_{0}^{\infty} \mathrm{d} \rho \int_{0}^{2 \pi} \mathrm{d} \phi \mathrm{e}^{i \omega r \sin \theta \cos (\varphi-\phi)} \\
& \times I_{N, S}(\rho) I_{N^{\prime}, S^{\prime}}(\rho) \mathrm{e}^{-i\left(l-l^{\prime}\right) \phi} \\
= & 2 \pi i^{l^{\prime}-l} \mathrm{e}^{i\left(l^{\prime}-l\right) \varphi}(-1)^{S+S^{\prime}} \\
& \times I_{S, S^{\prime}}\left(\frac{\omega^{2} \sin ^{2} \theta}{2 \mathrm{e} B}\right) I_{N^{\prime}, N}\left(\frac{\omega^{2} \sin ^{2} \theta}{2 \mathrm{e} B}\right) .
\end{aligned}
$$

\section{References}

Frangodimitraki-Georgiadou, M. 1991, Ph.D. Thesis, University of Tübingen

Baring, M., \& Harding, A. 1995, La Jolla Workshop on HVNS, in press

Burns, M., \& Harding, A. 1984, ApJ, 285, 747

Daugherty, J., \& Harding, A. 1983, ApJ, 273, 761

Harding, A. 1990, Proceedings of the International Conference on Coherent Radiation Processes in Strong Fields (The Catholic University)

Harding, A., \& Baring, M. 1997, ApJ, 476, 246

Semionova, L., \& Leahy, D. 1999, Phys. Rev. D, 60, 0733011

Semionova, L., \& Leahy, D. 2000, A\&AS, 144, 307

Sokolov, A., \& Ternov, I. 1983, Relativistic Electron (Nauka, Moscow) 